\title{
Is Liver Ultrasound Useful as Part of the Surveillance Strategy following Potentially Curative Colorectal Cancer Resection?
}

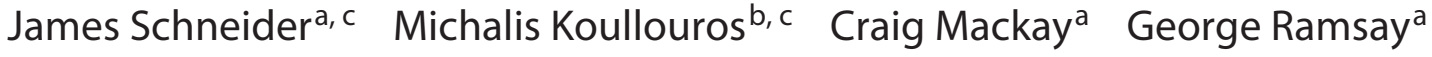 \\ Craig Parnaby ${ }^{a}$ Lynn Stevenson ${ }^{a}$ \\ ${ }^{a}$ Department of General Surgery, Aberdeen Royal Infirmary, Aberdeen, UK; ${ }^{b}$ Department of Mental Health, Basildon \\ University Hospital, Basildon, UK; ' University of Aberdeen, Aberdeen, UK
}

\section{Keywords \\ Colorectal cancer · Surveillance monitoring · Ultrasound}

\begin{abstract}
Background: Optimal surveillance monitoring following curative resection of colorectal cancer remains unclear. Guidelines recommend computed tomography (CT)-based imaging for the initial 3 years following surgical intervention due to the high rates of local and distant recurrence. However, there is currently limited supporting evidence for this strategy. Our current follow-up practice is to offer annual interval abdominal ultrasound and abdominal/pelvis CT scans starting at 6 and 12 months with the sequence of radiological follow-up remaining at the discretion of each clinician. We aim to establish the additional diagnostic benefit of abdominal ultrasound to CT scans in colorectal cancer surveillance follow-up. Methods: All patients who underwent colorectal resection with curative intent in our region during a single year were included. Patients were detected from a prospectively collected pathology database and supplemented retrospectively with patient demographics, imaging reports, and mortality data. Results: A total of 243 patients (male $n=135$, $55.6 \%)$ were included. There was a mortality rate of $31.3 \%$
\end{abstract}

\section{KARGER}

(c) 2018 S. Karger AG, Basel

E-Mail karger@karger.com

www.karger.com/ddi over the study period. Patients who received abdominal ultrasound as their initial imaging modality ( $n=64,26.3 \%$ ) were significantly older, had less severe disease, and a significantly lower mortality rate when compared to CT patients ( $n=148,60.9 \%$ ). All patients with new hepatic disease detected by ultrasound scans had their management discussed in multi-disciplinary team meetings before their next scheduled CT. Conclusion: In an era where cross-sectional imaging of colorectal cancer is commonplace, abdominal ultrasound offers additional benefit to CT as a postoperative imaging adjunct for the detection of hepatic disease recurrence.

(c) 2018 S. Karger AG, Basel

\section{Introduction}

Despite improvements in prevention, screening, investigations, and therapeutic measures, colorectal cancer is the second most common cause of cancer-related death in the United Kingdom in 2014 with approximately 1 death every $30 \mathrm{~min}$ [1] as a result of this disease. Approximately two thirds of patients have potentially resectable and therefore curable disease at diagnosis [2, 3]. However, malignancy recurrence in the form of local and distant
Dr. James Schneider

Department of General Surgery

Aberdeen Royal Infirmary

Aberdeen AB25 2ZD (UK)

E-Mail james.schneider@nhs.net 
disease is observed in $25-30 \%$ of cases $[4,5]$. These recurrences mostly occur in the liver, loco-regional anastomotic regions, and lungs, with metachronous liver lesions occurring in $15 \%$ of patients within 5 years $[6,7]$.

Current evidence confirms that early identification of local recurrence and/or metastases is associated with increased survival, especially if the patient is asymptomatic [8-10]. For this reason, postoperative surveillance is used to detect recurrence in its early stages. However, there is no consensus on the optimal surveillance-monitoring regimen.

The Scottish Intercollegiate Guidelines Network Guidelines recommend that interval computed tomography (CT) scanning could be beneficial for patients who undergo potentially curative colorectal resections [11]. Additionally, the National Institute for Health and Care Excellence suggests a minimum of $2 \mathrm{CT}$ scans within the first 3 years following surgical intervention [12]. Our current practice is to provide 3 years of imaging follow-up after resection with curative intent: CT chest, abdomen, pelvis and liver ultrasound scans (USS) are undertaken on an alternating basis 6 months apart for 3 years. Here, USS aim to expedite the detection of hepatic metastases, as the survival following liver resection is convincing with $38 \%$ surviving for 5 years [13]. The initial imaging methodology is dependent on the choice of the responsible clinician.

The efficacy of "intensive vs. non-intensive" surveillance monitoring with variable combinations of investigations has frequently been evaluated in systematic reviews and meta-analyses [10,14-17]. Intensive surveillance provides earlier detection of disease recurrence amenable to surgical treatment; however, the ultimate reduction in postoperative mortality from remains questionable. USS have been shown to have lower sensitivity in the detection of hepatic diseases compared to CT; however, the additional benefit to using USS in interval CT surveillance with respect to these clinical outcomes remains unclear $[18,19]$. Additional USS surveillance is also associated with increased costs and adds to further resource pressure.

We aim to determine if USS provide any additional benefit to interval yearly $\mathrm{CT}$ in the detection of hepatic disease in patients following potentially curative resection of colorectal cancer during postoperative surveillance.

\section{Methods}

\section{Patient Selection}

All patients who had resection of colorectal carcinoma with curative intent from January 2012 to December 2012 in NHS Grampian were included. Patients were excluded if they were known to have metastatic disease at diagnosis and the operation was performed for palliation. Those who had suffered a perioperative mortality or those with missing data regarding pathology and imaging reports were also excluded.

\section{Data Collection}

Pathological reports were prospectively maintained in a regional pathology database and this was supplemented retrospectively with imaging reports obtained from the picture archive and communication system, and demographic data, obtained from the local electronic patient records. Follow-up was established from a retrospective review of a weekly updated survival registry. All imaging had been reported by experienced radiologists, and any new findings were discussed at the colorectal cancer multi-disciplinary meeting. Reports were categorized into no significant findings, likely of local recurrence, distant metastatic disease, benign disease, and other primary malignancies. Positive findings were defined as those that were newly diagnosed (i.e., not detected by previous imaging during the surveillance period). Repeat positive results were subsequently excluded from data analysis for diagnostic yield. Emergency imaging for unrelated pathologies, such as appendicitis or biliary disease, which were not part of the surveillance program were excluded.

\section{Study Outcome Measures}

The primary outcome was the detection of new hepatic disease during our local surveillance-monitoring program. Secondary outcomes were changes to management after detection of new hepatic diseases and factors that influenced the choice of initial imaging modality.

\section{Statistical Analysis}

Statistical analysis was undertaken using SPSS version 23 (IBM, New York, NY, USA). Descriptive analysis was performed for population demographics and displayed in the form of frequency distribution tables for pathological features (location of tumour, DUKES Classification, and TNM Grading). Categorical data were analyzed by chi-square and nonparametric scale variables were analyzed by Kruskal-Wallis tests, with $p<0.05$ deemed as statistically significant. This was a retrospective observational review of current practice, which did not fulfill the requirements for ethical review.

\section{Results}

\section{Population Demographics and Pathological Features}

During the study period, 265 patients underwent colorectal resection with curative intent. A total of 6 patients were excluded due to early mortalities and 4 due to missing information (pathology and imaging reports). A further 12 patients were excluded due to the presence of metastatic disease (M1). Thus, the final study cohort comprised 243 patients (males $n=135$, $55.6 \%$ ), with a median age of 71.0 (IQR 79.0-63.7) years. Within the postoperative follow-up period, there were 76 deaths, a mortality rate of $31.3 \%$. Excluding patients 
who had a $<3$-year postoperative mortality $(n=53)$ or did not enter surveillance monitoring $(n=28)$, the median follow-up period was 36.3 (IQR 38.6-27.7) months. Additional pathological variables, including location, DUKES Classification and TNM Staging, are shown in Table 1. Overall, 1,025 scans were performed during the follow-up period, including 350 USS (34.1\%), and 605 CT (59.0\%) scans. Radiological findings from these imaging modalities can be found in Table 2 .

\section{Clinical Variables That Influenced the Choice of Initial Imaging}

In our surveillance protocol, the initial imaging modality is at the clinical discretion of the responsible clinician. Initial imaging postoperatively was predominantly CT $(n=148,60.9 \%)$ with 64 patients having USS $(26.3 \%)$. Subgroup analysis demonstrated that those who underwent CT imaging were younger $(p<0.001)$ and had higher DUKES Classification than those undergoing USS as first follow-up scan ( $p=0.05$; Table 3 ). They also had a higher rate of mortality (33.1\%), $p=0.019$. A total of 28 patients $(11.5 \%)$ did not undergo imaging in the postoperative period. This group had the most advanced age but did not differ greatly from those undergoing imaging in terms of pathological stage (Table 3 ). This group had the highest mortality rate $(p<0.05)$.

\section{Patient Management Post USS Detection of \\ Metastases}

Of the 12 patients who had new hepatic diseases detected by USS, the mean time to discussion at MDT after this new finding was 23 days. All had treatment decisions changed during this discussion: 4 patients had additional palliative chemotherapy, 5 patients were treated with best supportive care, and 3 patients were found to have benign disease but warranted further review. In this study, no cases were suitable for metastatectomy. All had their treatment altered before the interval CT was scheduled.

\section{Discussion}

This study aimed to review our experience with interval USS alongside CT-based surveillance monitoring following colorectal resections. Metachronous liver metastases account for a significant proportion of distant recurrence following surgical intervention. Early detection is fundamental in these patients, since the majority of these metastases will present within the first 2 years [20]. Furthermore, aggressive treatment of met-
Table 1. Pathological features

\begin{tabular}{lc}
\hline Pathological features & Frequency, $n(\%)$ \\
\hline Location of tumour & \\
Proximal & $99(40.7)$ \\
Distal & $83(34.2)$ \\
Rectal & $61(25.1)$ \\
DUKES classification & $39(16.0)$ \\
A & $101(41.6)$ \\
B & $89(36.6)$ \\
C1 & $13(5.3)$ \\
C2 & $1(0.4)$ \\
Missing data & \\
TNM staging & \\
T score & $17(7.0)$ \\
T1 & $31(12.8)$ \\
T2 & $133(54.7)$ \\
T3 & $62(25.5)$ \\
T4 & \\
N score & $140(57.6)$ \\
N0 & $68(30.0)$ \\
N1 & $35(14.4)$ \\
N2 & \\
M score & $243(100)$ \\
M0 &
\end{tabular}

Table 2. Overall diagnostic yield (NB - repeat positive results were excluded from data analysis for diagnostic yield, thus accounting for different denominator values)

\begin{tabular}{lcc}
\hline & $\begin{array}{c}\text { Abdominal } \\
\text { ultrasound, } n(\%)\end{array}$ & CT, $n(\%)$ \\
\hline No significant findings & $300 / 350(85.7)$ & $335 / 605(55.4)$ \\
Local recurrence & - & $27 / 520(5.2)$ \\
$\quad$ Liver & $12 / 302(4.0)$ & $25 / 361(6.9)$ \\
Lung & - & $21 / 361(5.8)$ \\
Other & $1 / 302(0.3)$ & $14 / 361(3.9)$ \\
Total & $13 / 302(4.3)$ & $51 / 361(14.7)$ \\
Benign pathology & $18 / 316(5.7)$ & $14 / 538(2.6)$ \\
Other malignancy & $0(0)$ & $5 / 594(0.8)$ \\
\hline
\end{tabular}

astatic lesions is available in the form of surgical resection or oncological therapies. The most appropriate method of performing surveillance monitoring, especially during these early years remains unclear. However, our data show that interval ultrasound was effective in detecting hepatic disease between annual CT scans in a total of 12 patients $(32.4 \%$ of patients who demonstrated evidence of metachronous hepatic recurrence) all of whom had clinical management changed as a result of this finding. 
Table 3. Clinical and pathological variables influencing initial choice of imaging

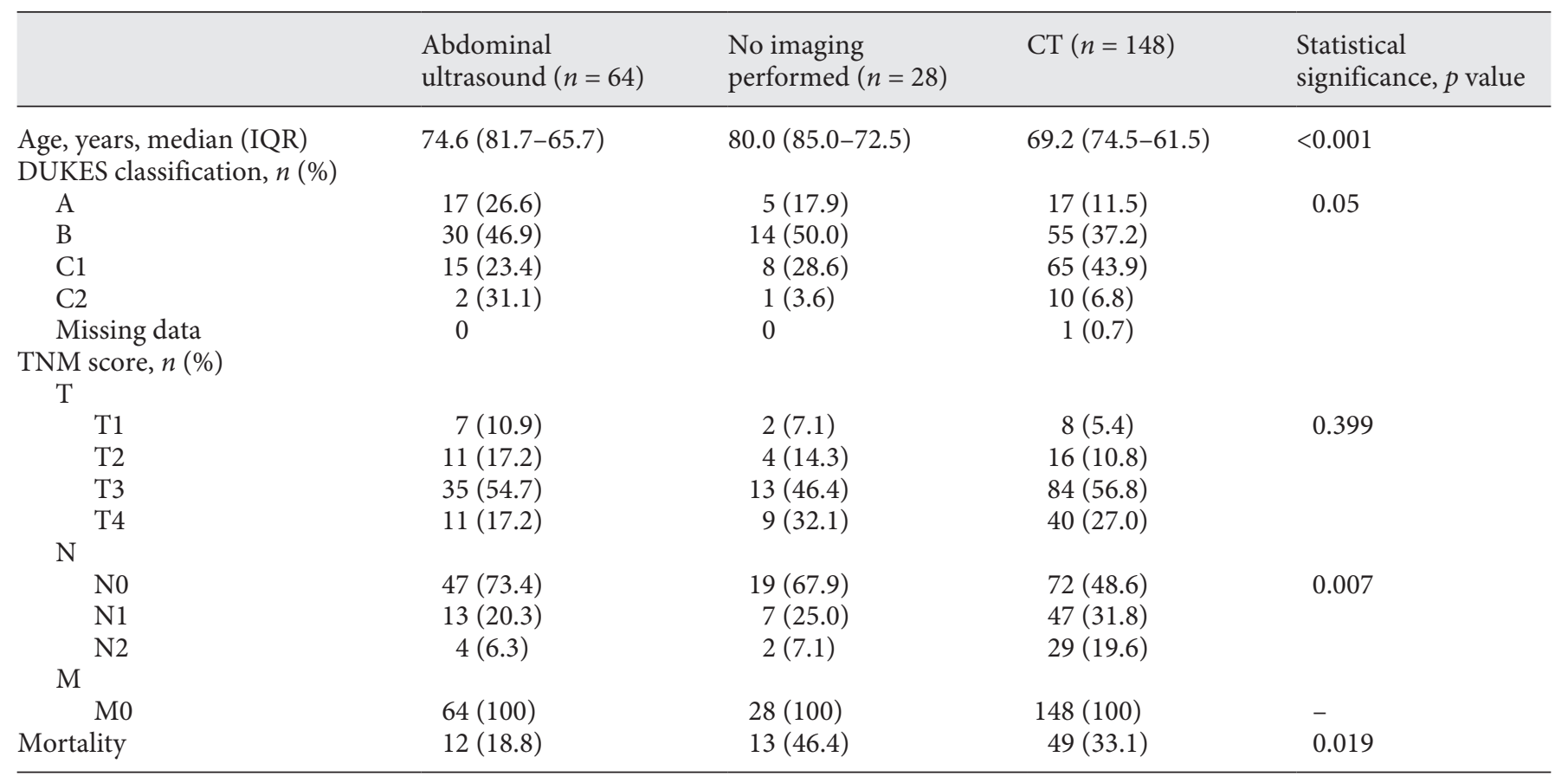

The multidisciplinary team provides an excellent service for risk stratification and identifying the most appropriate treatment for patients. However, it does not play a significant role with respect to surveillance monitoring. Therefore, initial imaging modality is dependent on clinician choice. Despite our local protocol suggesting that USS is the preferred initial imaging method in most cases, only $26.3 \%$ of patients in our study had USS as their first surveillance scan. The clinical features that differed between the subgroups could account for this difference in practice. Patients who had an initial follow-up USS were significantly older, had lower pathological stage of disease (with no previously identified metastatic disease), and greater survival rates. Thus, it appears that clinicians in our region already risk stratify before deciding on the appropriate radiological follow-up, despite what is dictated by local protocol.

Additionally, the patients with the poorest prognosis appear to be correctly identified. With careful discussion, those patients deemed not suitable for adjuvant therapies can be detected and prevented from being subject to regular investigations that will not alter their subsequent management. This emphasizes that clinical pragmatism in the era of realistic medicine remains an influential factor when deciding patient management. The retrospective nature of this study precludes us from analyzing this suspected clinical risk stratification in more detail. Other confounding factors involved in these decisions may have included the consideration of preexisting medical comorbidities and opinions expressed by the patient.

The prospective nature of the pathology database allowed an accurate representation of this population with minimal missing cases. Despite being limited by the retrospective data collection of other variables, this enabled us to audit our current practice. We recognize that 69 $(28.4 \%)$ patients were not strictly followed up for our lo$\mathrm{cal} \geq 3$ year surveillance-monitoring target. Unfortunately, due to the retrospective collection of data, we were unable to differentiate those who quit the program on personal/clinical grounds or were lost to follow-up. However, the majority of the study population were followed up for $\geq 2$ years in which the majority of malignancies are detected in surveillance monitoring [20].

On analysis of those 12 patients who had a diagnosis of hepatic disease detected by USS on this surveillance program, all had their management plans changed and, where appropriate, treatment started within 1 month of these findings. This expedited the care options available to these patients by detecting their recurrent malignancy earlier than would have been the case in a follow-up strategy of annual CT scans. In this particular cohort, unfortunately all malignant cases were for palliative treatment. 
However, this early detection may indicate the potential for identification of cases for more aggressive metastatectomy resections before further spread had occurred.

Our data suggests that USS could be considered a valuable imaging modality alongside annual CT surveillance for colorectal cancer patients. Ultrasound is a painless, safe, and relatively cheap imaging technique and this study demonstrates that it can be an effective adjunct to $\mathrm{CT}$ in detecting metastatic colorectal cancer in its early stages. Additional scans to those suggested in the national institute for health and care excellence and Scottish intercollegiate guidelines network guidelines will increase radiological workload and therefore impact on service demand. However, detecting metastatic deposits early is ever more relevant, given the extensive treatment strategies available, including those aiming at cure for this patient group.

\section{Disclosure Statement}

This work has not been published and is not being considered for publication elsewhere. The authors of this paper do not have any conflicts of interest. This study was conducted within the General Surgery Department at the Aberdeen Royal Infirmary. During the data collection period, 2 authors were affiliated with the University of Aberdeen.

\section{References}

1 Cancer Research UK. Bowel Cancer Mortality Statistics. Cancer Res UK, 2014.

2 Safi F, Beyer HG: The value of follow-up after curative surgery of colorectal carcinoma. Cancer Detect Prev 1993;17:417-424.

3 Phillips RK, Hittinger R, Blesovsky L, Fry JS, Fielding LP: Local recurrence following "curative" surgery for large bowel cancer: II. The rectum and rectosigmoid. Br J Surg 1984;71: $17-20$.

4 Olson RM, Perencevich NP, Malcolm AW, Chaffey JT, Wilson RE: Patterns of recurrence following curative resection of adenocarcinoma of the colon and rectum. Cancer 1980;45: 2969-2974.

5 Bohm B, Schwenk W, Hucke HP, Stock W: Does methodic long-term follow-up affect survival after curative resection of colorectal carcinoma? Dis Colon Rectum 1993;36:280286.

6 Manfredi S, Lepage C, Hatem C, Coatmeur O, Faivre J, Bouvier AM: Epidemiology and management of liver metastases from colorectal cancer. Ann Surg 2006;244:254-259.

7 Sadahiro S, Suzuki T, Ishikawa K, Nakamura T, Tanaka Y, Masuda T, et al: Recurrence patterns after curative resection of colorectal cancer in patients followed for a minimum of ten years. Hepatogastroenterology 2003;50: $1362-1366$.
8 Rodriguez-Moranta F, Salo J, Arcusa A, Boadas J, Pinol V, Bessa X, et al: Postoperative surveillance in patients with colorectal cancer who have undergone curative resection: a prospective, multicenter, randomized, controlled trial. J Clin Oncol 2006;24:386-393.

9 Rosen M, Chan L, Beart RW Jr, Vukasin P, Anthone G: Follow-up of colorectal cancer: a meta-analysis. Dis Colon Rectum 1998;41: 1116-1126.

10 Pita-Fernandez S, Alhayek-Ai M, GonzalezMartin C, Lopez-Calvino B, Seoane-Pillado T, Pertega-Diaz S: Intensive follow-up strategies improve outcomes in nonmetastatic colorectal cancer patients after curative surgery: a systematic review and meta-analysis. Ann Oncol 2015;26:644-656

11 Scottish Intercollegiate Guidelines Network (SIGN). Diagnosis and management of colorectal cancer. SIGN Guidel 2011;126:11.

12 National Institute for Health and Care Excellence (NICE). Colorectal Cancer: Diagnosis and Management, 2011.

13 Kanas GP, Taylor A, Primrose JN, Langeberg WJ, Kelsh MA, Mowat FS, et al: Survival after liver resection in metastatic colorectal cancer: review and meta-analysis of prognostic factors. Clin Epidemiol 2012;4:283-301.

14 Mokhles S, Macbeth F, Farewell V, Fiorentino F, Williams NR, Younes RN, et al: Meta-anal- ysis of colorectal cancer follow-up after potentially curative resection. Br J Surg 2016; 103:1259-1268.

15 Renehan AG, Egger M, Saunders MP, O'Dwyer ST: Impact on survival of intensive follow up after curative resection for colorectal cancer: systematic review and meta-analysis of randomised trials. BMJ 2002;324:813.

16 Tjandra JJ, Chan MK: Follow-up after curative resection of colorectal cancer: a metaanalysis. Dis Colon Rectum 2007;50:17831799.

17 Jeffery M, Hickey BE, Hider PN: Follow-up strategies for patients treated for non-metastatic colorectal cancer. Cochrane Database Syst Rev 2007;1:CD002200.

18 Glover C, Douse P, Kane P, Karani J, Meire H, Mohammadtaghi S, et al: Accuracy of investigations for asymptomatic colorectal liver metastases. Dis Colon Rectum 2002;45:476-484.

19 Kinkel K, Lu Y, Both M, Warren RS, Thoeni RF: Detection of hepatic metastases from cancers of the gastrointestinal tract by using noninvasive imaging methods (US, CT, MR imaging, PET): a meta-analysis. Radiology 2002; 224:748-756.

20 Griffin MR, Bergstralh EJ, Coffey RJ, Beart RW Jr, Melton LJ 3rd: Predictors of survival after curative resection of carcinoma of the colon and rectum. Cancer 1987;60:2318-2324. 